\title{
PLEOMORPHIC UNDIFFERENTIATED SARCOMA OF THE PENIS
}

doi: $10.1590 / \mathbf{S 1 8 0 7 - 5 9 3 2 2 0 0 8 0 0 0 6 0 0 0 2 2}$

Omar Reda El Hayek, ${ }^{\mathrm{I}}$ Roberto Ibrahim, ${ }^{\mathrm{II}}$ Gustavo Caserta Lemos ${ }^{\mathrm{II}}$

\section{INTRODUTION}

Primary penile sarcoma is an extremely rare condition. The incidence of penile sarcoma is described as 0.6 to 1 out of 100,000 patients in developed countries, and leiomyosarcoma is the predominant histological type. ${ }^{1}$ Given such a low incidence, treatment for this condition is very controversial. The authors could not find in the literature any other case of penile sarcoma with such histological findings (Pleomorphic undifferentiated sarcoma).

\section{CASE REPORT}

J.M. is a 78 year-old male who was experiencing discomfort and pain in the perineal region for one month. Physical examination revealed a 5 centimeter perineal nodule right below the scrotum. The ultrasound examination showed a solid mass with intense vascularization arising from the corpora cavernosa, while the MRI showed no evidence of invasion into adjacent structures (Figure 1). Core biopsy of the lesion revealed a pleomorphic undifferentiated sarcoma originating from the corpora cavernosa. Abdominal and chest work-ups were negative. The patient was submitted to neoadjuvant chemotherapy with Doxorubicin $\left(25 \mathrm{mg} / \mathrm{m}^{2} /\right.$ day $)$ and Iphosphamide $\left(1.7 \mathrm{mg} / \mathrm{m}^{2} /\right.$ day) from D1 to D3, followed by intensity-modulated radiotherapy (total dose of $66 \mathrm{~Gy}$ ) of the pelvic area, lymph nodes, and the base of the corpora cavernosa. The result demonstrated good response and partial regression of the mass. The patient was then submitted to total penectomy, as well as intraoperatory radiation at a dose of $6 \mathrm{MeV}$ of electrons and $10 \mathrm{~Gy}$, with a $51 \mathrm{~mm}$ cone.

\footnotetext{
I Department of Urology, Hospital das Clinicas, Faculdade de Medicina, Universidade de São Paulo - São Paulo/SP, Brazil.

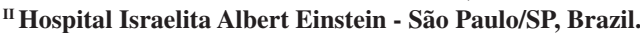

Email: hayek@uol.com.br

Tel.: 55119975.8699
}
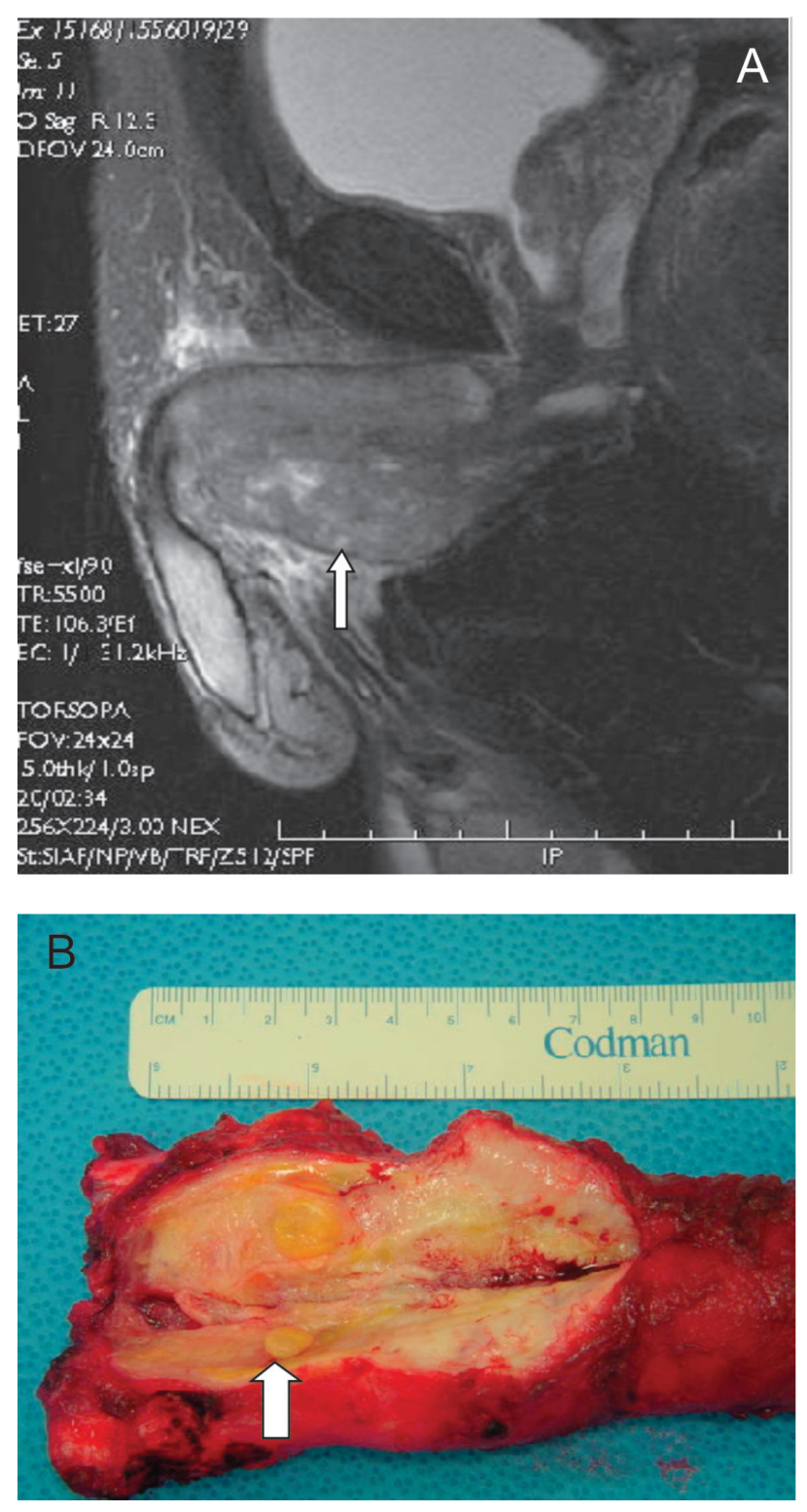

Figure 1A - MRI. The arrow shows deep infiltration into the corpora cavernosa. 1B- Total penectomy specimen showing extensive penile infiltration 
Histopathological findings revealed a pleomorphic, highgrade sarcoma and negative margins upon resection (Figure 2A). All immunohistochemical panels were negative (Figure 2B). Four months later, the patient presented inguinal pain and a CT scan showed a lymph node mass of $2.4 \mathrm{~cm}$. The mass was promptly removed, revealing the presence of an undifferentiated sarcoma invading the adjacent fat tissue. The patient received an extra course of chemotherapy consisting of Taxotere at $36 \mathrm{mg} / \mathrm{m}^{2}$ and Gencitabine at 800
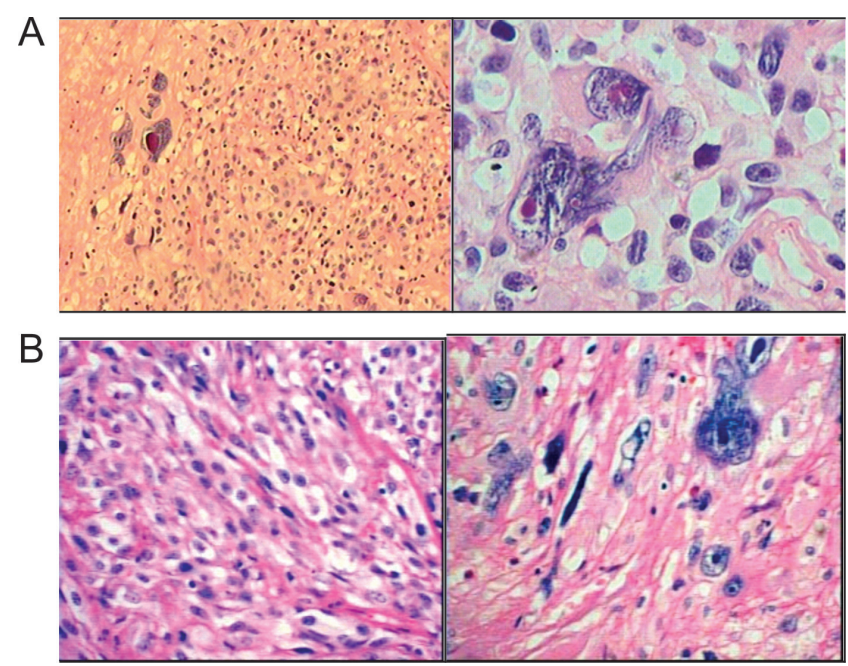

Figure 2A - Undifferentiated Pleomorphic Sarcoma of the penis (HE, X100). B- Immunohistochemical panels, negative for all tested antibodies (CD117- CKit, HHF35, Desmin, CD34-KBEND10, CD31- JC70A, F100 protein, AE1AE3, EMA and CD20, 45, 30) $\mathrm{mg} / \mathrm{m}^{2}$ from D1-D8. Systemic progression occurred during the chemotherapy, with the appearance of pulmonary and hepatic nodules. The patient was then administered Thalidomide with no response, and died from the disease 17 months after the initial diagnosis.

\section{DISCUSSION}

Primary sarcoma of the penis is a very rare condition, and to date there are only around 44 cases described in the medical literature, of which 30 are leiomyosarcomas and 14 are epithelial sarcomas. ${ }^{2}$ Different types of sarcomas are even rarer and, after extensive literature searches, we were unable to find any other cases of primary pleomorphic undifferentiated sarcoma of the penis. One possible explanation for these histological findings could be the occurrence, at some point, of a de-differentiation. On the other hand, the tumor could have been pleomorphic from the start. ${ }^{3}$

The therapeutic approach to such cases is very challenging and remains controversial. We do know that these lesions originate from the base of the corpora cavernosa and are usually treated by total penectomy with or without associated radiation and chemotherapy. ${ }^{3}$ Regardless of the therapeutic modality used, the majority of patients die in less than two years due to systemic dissemination ${ }^{3}$. The most appropriate approach to treating such conditions remains to be determined.

\section{REFERENCE}

1. Isa SS, Almaraz R, Magovern J. Leiomyosarcoma of pênis: case report and review of the literature. Cancer. 1984;54:939-42.

2. Fetsch JF, Davis CJ, Miettine M, Sesterhenn IA. Leiomyosarcoma of the penis: A clinicopathological study of 14 cases with review of the literature and discussion of the differential diagnosis. Am J Surg Pathol. 2004;28:115-25.
3. Antunes AA, Nesrallah LJ, Gonçalves PD, Ferreira YA, Campagnari JC Srougi M. Deep-seated sarcomas of the penis. Int Braz J Urol. 2005;31:245-50. 